\title{
Erratum: Nonlinear dynamics of a self-mixing thin-slice solid-state laser subjected to Doppler-shifted optical feedback [Phys. Rev. E 104, 044203 (2021)]
}

\author{
Kenju Otsuka $\odot$ and Seiichi Sudo $\odot$
}

Q (Received 23 November 2021; published 9 December 2021)

DOI: 10.1103/PhysRevE.104.069901

This article contains two typographical errors.

The first one is located in the caption of Fig. 4(c). The base of the logarithmic function for the soft mode should read $e$ (Euler's number).

The second typographical error is located in the descriptive formula of the spontaneous emission coefficient $\varepsilon$, given in the second paragraph of Sec. IV C. It should read $\varepsilon=c \sigma \tau / \pi w_{o}^{2} n L$.

All calculations and figures in the paper were performed with the correct expressions above, and none of the results presented in the paper are affected by the typographical errors. 